\title{
A Rare Cause of Dyspnea in SARS-CoV-2 Emergency: A Case of Cardiac Tamponade During Pembrolizumab Immunotherapy for Lung Cancer
}

\author{
Andrea Vergara' ${ }^{1,2 *}$, Marco De Felice ${ }^{3}$, Felice Gragnano ${ }^{1,2}$, Arturo Cesaro ${ }^{1,2}$, Elisabetta \\ Moscarella $^{1,2}$, Fabio Fimiani, ${ }^{4}$ Davide Leopardo ${ }^{5}$, Giovanni Pietro Ianniello ${ }^{5}$ and Paolo \\ Calabrò ${ }^{1,2}$ \\ ${ }^{1}$ Department of Translational Medical Sciences, University of Campania 'Luigi Vanvitelli', Caserta,Italy
}

${ }^{2}$ Division of Clinical Cardiology, Cardiovascular Department, A.O.R.N. 'Sant'Anna e San Sebastiano', Caserta, Italy

${ }^{3}$ Department of Precision Medicine, University of Campania "Luigi Vanvitelli", Caserta, Italy

${ }^{4}$ Unit of Inherited and Rare Cardiovascular Diseases, AORN dei Colli “V. Monaldi", Naples, Italy

${ }^{5}$ Division of Oncology, A.O.R.N 'Sant'Anna e San Sebastiano', Caserta, Italy

*Corresponding author: Andrea Vergara, Division of Clinical Cardiology, A.O.R.N. Sant'Anna e San Sebastiano, Italy, University of Campania “Luigi Vanvitelli", Italy.

To Cite This Article: Andrea Vergara, Marco De Felice, Felice Gragnano, Arturo Cesaro, Elisabetta Moscarella, et al., A Rare Cause of Dyspnea in SARS-CoV-2 Emergency: A Case of Cardiac Tamponade During Pembrolizumab Immunotherapy for Lung Cancer. Am J Biomed Sci \& Res. 2021 14(1). AJBSR.MS.ID.001956. DOI: 10.34297/AJBSR.2021.14.001956.

Received: 韮 August 24,2021; Published: 割 September 02, 2021

\begin{abstract}
Pembrolizumab - an immune checkpoint inhibitor (ICI) - is commonly used for immunotherapy in patients with lung cancer. Unlike other anti-cancer agents, ICIs can produce specific immune-related adverse events that can affect cardiovascular system in approximately $1 \%$ of cases. Cardiovascular complications include more often heart failure, dilated cardiomyopathy, myocarditis, and conduction abnormalities. Pericardial effusion and cardiac tamponade after ICI therapy are rare and due to acute lymphocytic pericarditis. In patients with cardiac tamponade, clinical presentation can be featured by fever and acute dyspnea, potentially mimicking acute respiratory disease such as SARS-CoV-2 infection and complicating their management. We report the first case of cardiac tamponade due to pembrolizumab in a patient with lung adenocarcinoma admitted to the emergency department during the COVID-19 pandemic.
\end{abstract}

Keywords: cardiooncology; cardiac tamponade; immunotherapy

\section{Introduction}

A 60-year-old man, heavy smoker, with an established diagnosis of lung adenocarcinoma (cT3N2M1b) treated with pembrolizumab was admitted to the emergency department with fever, acute dyspnoea, and cough. The clinical examination revealed unstable hemodynamic status and respiratory failure, featured by hypotension (systolic pressure 80/50 $\mathrm{mmHg}$ ), tachycardia (heart rate $120 \mathrm{bpm}$ ), and hypoxia (Sp02 78\%). Because COVID-19 pandemic, the patient was suspected of SARS-CoV-2 infection and therefore isolated. According to the Institution protocol, he received a rhino- and oro-pharyngeal swab for SARS-CoV-2 RNA on admission and was referred for emergent computer tomography (CT) showing large pericardial effusion (figure 1 and figure 2), right pleural effusion, and concomitant progression of the lung cancer lesions. Cardiac ultrasound confirmed CT views, revealing cardiac tamponade and right ventricle collapse. An emergent pericardiocentesis was performed and about $800 \mathrm{ml}$ of bloody effusion were removed, with a substantial improvement of respiratory symptoms and oxygen saturation. A thoracentesis was also performed for diagnostic purposes. At 4 hours from admission, the results of the SARS-CoV-2 test was available and negative for viral RNA. Pericardial and pleural cytological examinations identified thyroid transcription factor 1 (TTF-1) positive malignant 
cells, suggesting a progression of lung cancer. Carcinoembryonic antigen (CEA) levels were above $200 \mathrm{ng} / \mathrm{ml}$ in the pericardial fluid and $500 \mathrm{ng} / \mathrm{ml}$ in the pleural fluid. After careful discussion between cardiologist, onclologists, and pneumologists, pembrolizumab therapy was deemed the culprit of both cancer (hyper)-progression and cardiac tamponade.

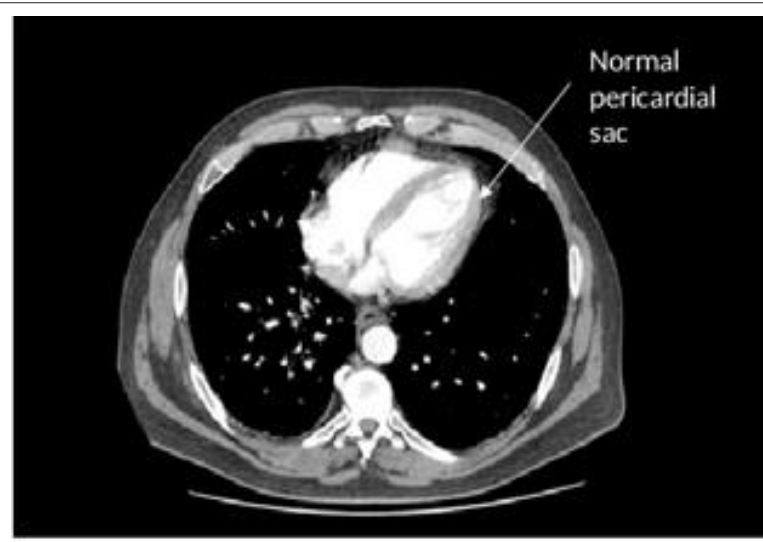

Figure 1: Contrast-enhanced chest computed tomography scan before pembrolizumab therapy.

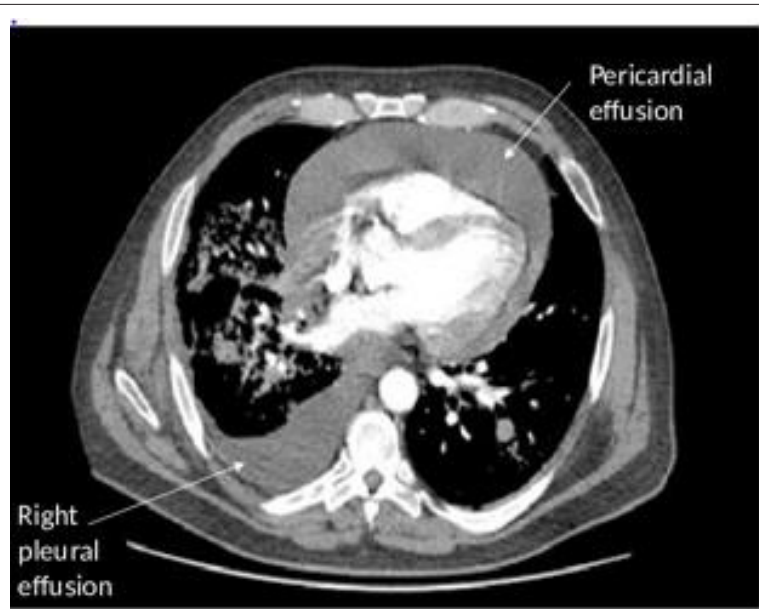

Figure 2: Contrast-enhanced chest computed tomography scan after pembrolizumab immunotherapy in emergency department.

Therefore, the drug was discontinued, and methylprednisolone at doses of $0.5 \mathrm{mg} / \mathrm{kg}$ was initiated to improve respiratory symptoms. Second-line treatment with carboplatin and pemetrexed was prescribed two weeks later. After two months, the patient was asymptomatic and the lung cancer had not progressed.

\section{Discussion}

In the emergency department, the management of patients with dyspnea remains critical, as it may have cardiac, respiratory, neurological, or psychogenic origin. Although during the COVID-19 pandemic, patients admitted with respiratory symptoms are primarily suspected for SARS-CoV-2 infection, dyspnea from cardiac causes remains frequent and should be carefully assessed to avoid rapid worsening of patient's condition. Cardiac tamponade is a relatively rare cause of dyspnea and in most cases idiopathic, but should be suspected of specific origin in cancer patients treated with immunotherapy [1]. Since initial investigations, somewhat unconventional patterns of response have been observed in patients on immunotherapy with ICIs compared with chemotherapy or target therapies, including hyper-progression of cancer disease.

Hyper-progression represents a paradoxical acceleration of the disease during immunotherapy with a rapid worsening of the patient's clinical conditions [2]. The actual incidence of immunerelated pericarditis in patients with advanced lung cancer in clinical practice remains unclear. A specific association between pericardial disease and lung cancer has been proposed [3], and Canale et al. reported pericardial effusion in approximately $7 \%$ of patients during immunotherapy treatment (with or without concomitant pleural effusion) [4]. In most cases, pericardial effusion is relatively mild, occurs within the first 3 months from immunotherapy initiation, and can be treated with corticosteroids as other immune-related adverse events [5]. The cytological examination usually reveals no malignant cells in the pericardial fluid [6], and the etiological diagnosis is often hard to define in these patients.

A case of cardiac tamponade during pembrolizumab for advanced lung adenocarcinoma has been previously reported [7]. However, pericardial effusion developed slowly and after a relatively long treatment period (i.e., four cycles). Therefore, pembrolizumab was safely continued after successful pericardiocentesis [7].

With respect to previous reports, our case is unique and substantially different as our patient experienced a rapid progression of cancer disease - within less than 2 months of immunotherapy - with a clear increase in the number and size of lung lesions. Also, cardiac tamponade and pleural effusion developed rapidly, resulting in acute clinical worsening and admission to the emergency department. CT imaging and pericardial/pleural effusion examination confirmed the hyper-progression of lung adenocarcinoma under pembrolizumab immunotherapy that was promptly diagnosed and managed thanks to the prompt and effective collaboration of a multi-disciplinary team composed by cardiologists, oncologists and pneumologists.

\section{Conclusion}

We report the first case of ICI-related cardiac tamponade in a patient treated with pembrolizumab for lung cancer during the COVID-19 pandemic. Cardiac tamponade and rapid cancer progression are rare complications of pembrolizumab immunotherapy. During the COVID-19 pandemic, cancer patients presenting with dyspnea should be rapidly referred for an expert 
and multidisciplinary evaluation to consider possible cardiac and/ or pulmonary complications secondary to primary cancer and concomitantanti-cancer treatment (figure 3). In this context, cardiooncology units should be implemented for a prompt evaluation of patients presenting with respiratory failure to guarantee proper and timely management of this special population during the COVID-19 pandemic.

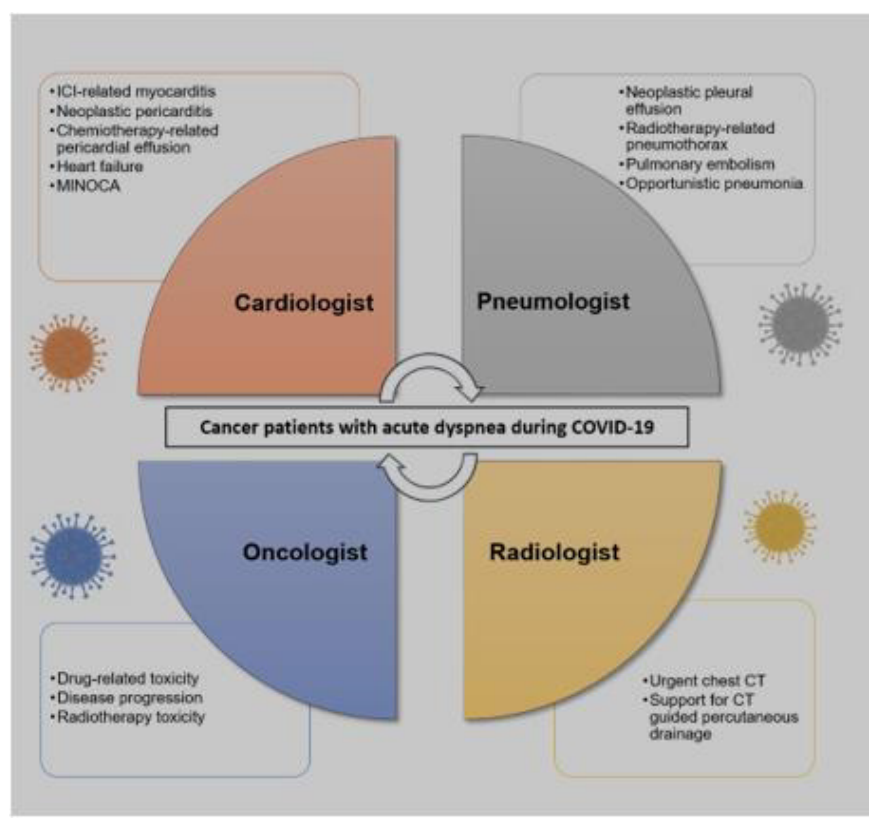

Figure 3: Management flow chart of cancer patients with acute dyspnea during COVID-19.

\section{Conflict of interests}

The authors declare that they have no known competing financial interests or personal relationships that could have appeared to influence the work reported in this paper.

\section{Acknowledgement}

None.

\section{References}

1. Sagrista Sauleda J, Merce J, Permanyer Maralda G, J Soler Soler (2000) Clinical clues to the causes of large pericardial effusions. Am J Med 109(2): 95-101.

2. Champiat S, Besse B, Marabelle A (2019) Hyperprogression during immunotherapy: do we really want to know? Ann Oncol 30(7): 10281031.
3. Salem J E, Manouchehri A, Moey M, B L Vignes, Lisa B, et al. (2018) Cardiovascular toxicities associated with immune checkpoint inhibitors: an observational, retrospective, pharmacovigilance study. Lancet Oncol 19(12): 1579-1589.

4. Canale M L, Camerini A, Casolo G, Lilli A, Bisceglia I, et al. (2020) Incidence of Pericardial Effusion in Patients with Advanced Non-Small Cell Lung Cancer Receiving Immunotherapy. Adv Ther 37(7): 3178-3184.

5. Shaheen S, Mirshahidi H, Nagaraj G, Hsueh C T (2018) Conservative management of nivolumab-induced pericardial effusion: A case report and review of literature. Exp Hematol Oncol 7(11): 2-5.

6. Kushnir I, Wolf I (2017) Nivolumab-induced pericardial tamponade: A case report and discussion. Cardiology 136(1): 49-51.

7. Harada K, Ogasawara M, Shido A, Akimitsu M, Soichiro O, et al. (2020) Pericardial tamponade during pembrolizumab treatment in a patient with advanced lung adenocarcinoma: A case report and review of the literature. Thorac Cancer 11(5):1350-1353. 